Vol. 4, Special Issue 2, January 2017

\title{
Press Machine Automation
}

\author{
Gorave Aparna ${ }^{1}$, Joshi S.S ${ }^{2}$ \\ Student E\&TC Department, Dr. Daulatrao Aher College of Engineering, Karad, India ${ }^{1}$ \\ Assistant Prof. E\&TC Department, Dr. Daulatrao Aher College of Engineering, Karad, India ${ }^{2}$
}

\begin{abstract}
An insertion of sheet metal in a press machine is a major problem in a kalburgi industry. To overcome the problem by our project" Press machine automation with AVR control." There is rapid need of automation reduce human effort and increase the work efficiency, here we are going to design such a system which automatically manage and control the punching operation of sheet metal by using AVR controller This device offer a unique combination of performance power efficiency and design flexibility. AVR (automatic voltage regulator) is a hardware device used to maintain voltage to electronic device. Inductive proximity sensor used for the detection of metal object. When a metal object is placed within the magnetic field generated by the sensor, the resulting current induced from an additional load and the oscillation ceases. This Causes the output driver to operate and depending on the normally open and, normally close the output signal is produced .In this way to detect a sheet metal this is the main aim of the project.
\end{abstract}

Keywords: Punching Force, AVR controller, Manufacturing Process.

\section{INTRODUCTION}

The $21^{\text {st }}$ century is said to be century of inventions, automation. Now, a days automation plays important role in industry. Automatic control system, which saves manpower and financial statement\& provide better accuracy. Hence we have selected the project "Press Machine Automation."

Now a days in industries especially in automobile and other industries the automatic sheet metal press machine. Earlier the press machine where operated manually so the output of machine was very less so there is need to be an automation. The main aim and concept of this project is to have the overcome the problem metal sheet insertion in press machine by using the automation this problem will be reduced because automation saves the time, it saves the energy, it reduces the labour efforts, it maintains the accuracy, and provide good quality of product (Programmable Logic Controller) becomes more expensive project. With Raspberry Pi also increases more complexity for project. It is like mini- computer. Using Raspberry Pi, reboot the system every time and these not possible to reboot every time. So we have to design system with AVR. Maintains the accuracy and provide good quality of product the design and fabrication of automatic punching machine controlled by PLC this process can have a greater control over the process [1].

\section{PROPOSED SYSTEM}

\section{A. Block Diagram Description}

$12 \mathrm{~V}$ 10A SMPS power supply is required for project. Proximity Sensor is a sensor able to detect presence of nearby objects without any physical contact. Proximity sensors emits electromagnetic field or beam of electromagnetic radiation and looks for changes in field or return signal.

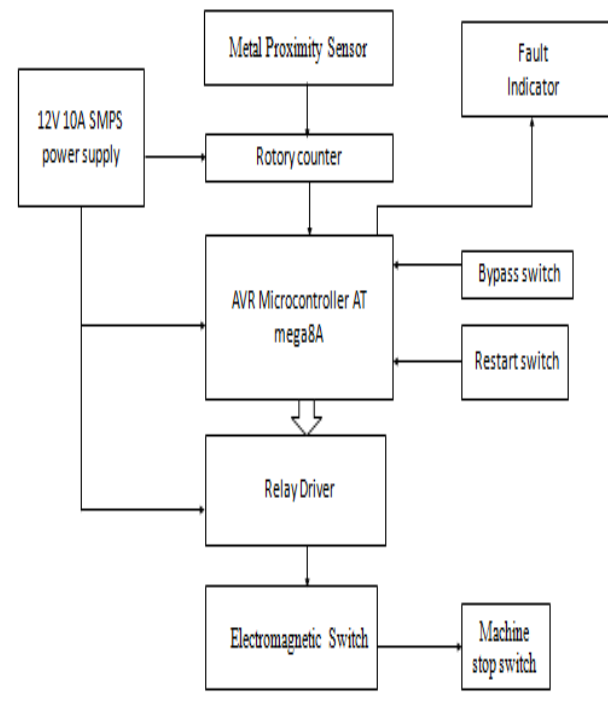

Fig.1.Proposed System

The object being sensed is referred to proximity sensors target. Proximity sensor can have high reliability and long functional life because of absence of mechanical parts and lack of physical contacts between sensed objects.

AVR (Automatic Voltage Regulator) is a hardware device used to maintain voltage to electronic device. Atmel AVR core combines instruction set with 32 general purpose working register. These directly connected to ALU allow him to independent registers to be accessed in one single inst. executed in one clock cycle.AVR uses Harvard architecture with separate memories and buses for programming data. Atmel AVR core combines a rich instruction set with 32 general purpose working registers. All the 32 registers are directly connected to the Arithmetic Logic Unit. 
Quad Relay driver used to increases current limit. Relay is relay between the two circuits; the link is magnetic and an electromagnetic switch which is useful if you want to mechanical. The coil of a relay passes a relatively large use a low voltage circuit to switch on and off a light current, typically $30 \mathrm{~mA}$ for a $12 \mathrm{~V}$ relay, but it can be as connected to a circuit.

Output of micro-controller is not sufficient to drive the voltages

relay directly. Therefore to drive the relay we are using Fault indicator is a device which provides visual indication relay driver block by using transistor as switch. A relay is of fault on the electric power system also called a faulted an electrically operated switch. Current flowing through circuit indicator, the device is used in electric power the coil of the relay creates a magnetic field, which attracts distribution network as a means of automatically detecting a lever and changes the switch contacts. The coil current and identifying faults to reduce outage time.

can be on or off so relays has two switch positions and Bypass switch is a hardware device that provides a failthey are double throw (changeover) switches. Relays safe access port for an in line active security appliance, it allow one circuit to switch a second circuit, which can be keeps network traffic flowing when the in line appliance completely separate from the first. For example a low fails if a link goes down, the bypass switch immediately voltage battery circuit can use a relay to switch a $230 \mathrm{~V}$ AC switches into bypass mode.

mains circuit. There is no electrical connection inside the

\section{FLOWCHART}

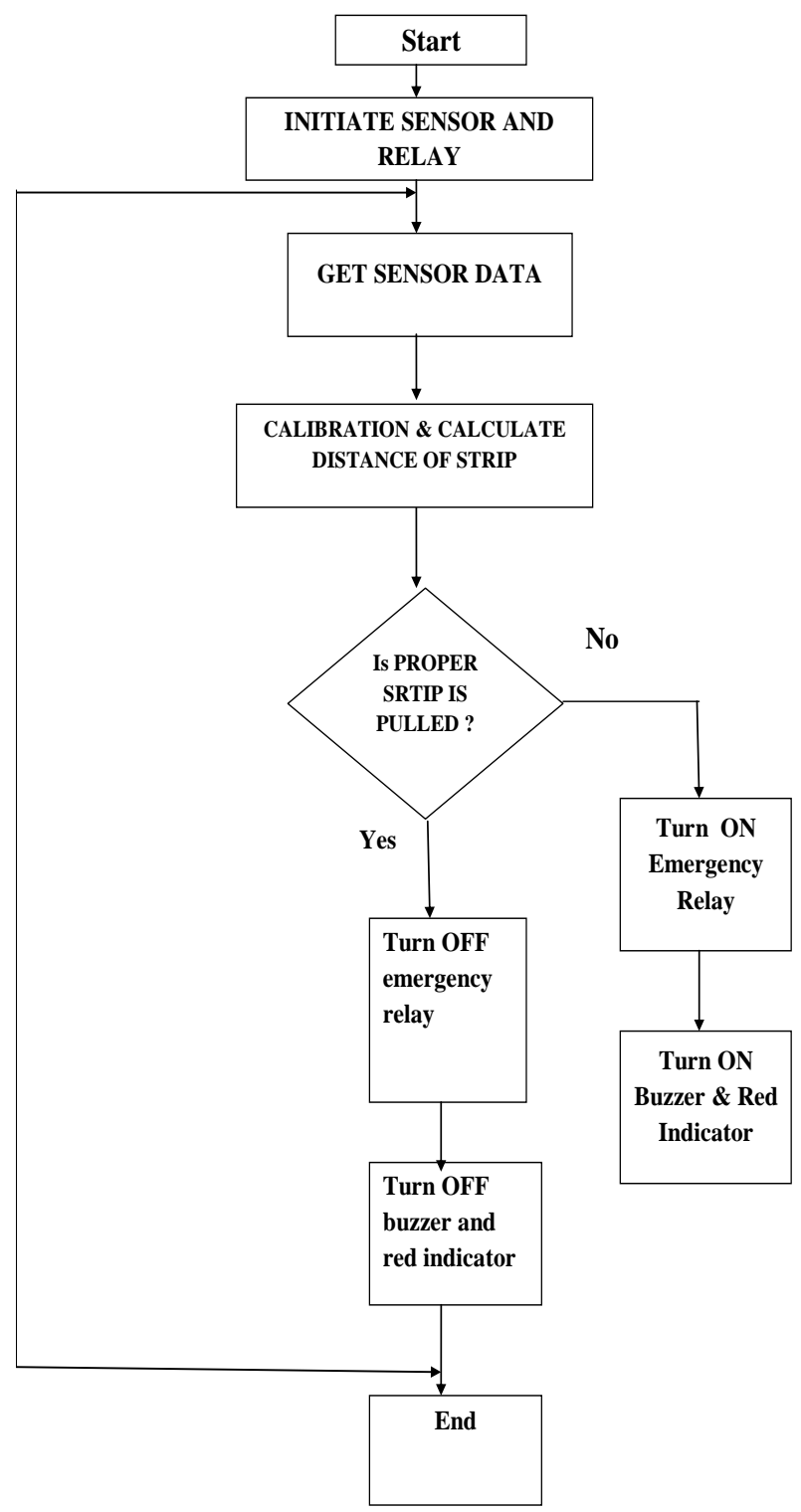

Fig .2.Flow Chart 


\section{FLOW CHART DESCRIPTION}

The machine starts when supply pass through the machine then initiate sensor and relay .If the strip inserted into machine is sensed by a metal proximity sensor then calibration and calculate the distance of strip if the strip pulled properly then turn off emergency relay and buzzer otherwise if any error occurred then turn on emergency relay and buzzer.

\section{HARDWARE REQUIREMENTS}

AVR Microcontroller-ATmega8A

The Atmel ATmega8A is a low-power CMOS 8-bit microcontroller based on the AVR enhanced RISC architecture. By executing powerful instructions in a single clock cycle, the ATmega8A achieves throughputs close to 1MIPS per MHZ. This empowers system designer to optimize the device for power consumption versus processing speed. The Atmel AVR core combines a rich instruction set with 32 general purpose working registers. All the 32 registers are directly connected to the Arithmetic Logic Unit (ALU), allowing two independent registers to be accessed in one single instruction executed in one clock cycle. The resulting architecture is more code efficient while achieving throughputs up to ten times faster than conventional CISC microcontrollers.

The ATmega8A provides the following features: $8 \mathrm{~K}$ bytes of In-System Programmable Flash with Read- WhileWrite capabilities, 512 bytes of EEPROM, $1 \mathrm{~K}$ byte of SRAM, 23 general purpose I/O lines, 32 general purpose working registers, three flexible Timer/Counters with compare modes, internal and external interrupts, a serial programmable USART, one byte oriented Two-wire Serial Interface, a 6- channel ADC (eight channels in TQFP and QFN/MLF packages) with 10-bit accuracy, a programmable Watchdog Timer with Internal Oscillator, an SPI serial port, and five software selectable power saving modes.

\section{Metal Proximity Sensor}

Proximity sensors are discrete sensors that sense when an object has come near to the sensor face there are 4 fundamental types of proximity sensors the inductive proximity sensor ,capacitive proximity sensor ,ultrasonic proximity sensor and optical proximity sensor.

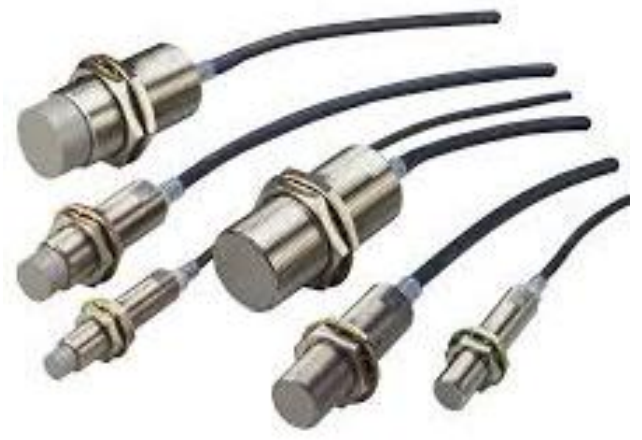

Fig .3. Metal Proximity Sensor
In order to properly specify and apply proximity sensor ,it is important to understand how they operate and to which application each is best suited. Inductive proximity sensor detect the presence of metal objects which come within range of their oscillating field and provide target detection to zero speed .Internally an oscillator creates high frquency electromagnetic field which is radiated from the coil and out from the sensor

Inductive proximity sensor detect the presence of metal objects which come within range of their oscillating field and provide target detection to zero speed .Internally an oscillator creates high frquency electromagnetic field which is radiated from the coil and out from the sensor face.

Selection of the proper proximity sensor depends on the size, material,and spacing of the target being sensed and the sensing distance that can be maintained.

The maximum sensing distance is defined as the distance in which the sensor is just closed enough to detect a ferrous target whose diameter is equl to or greater than sensor diameter in actual application the sensing distance should be between 50 to $80 \%$ of the maximum sensing range to assure reliable detection.For target size smaller than sensor diameter the maximum sensing distance can be estimated. Inductive proximity sensor are available in both DC and AC power model most required three electrical connection -ground,power ,output .However there are other variations that requires two wires and four wires .most sensors are available with a built in LED that indicates when the sensor output is on.

\section{Relay Driver}

A relay is an electromagnetic switch by a relatively small electric current that can turn on or off a much larger electric current. The heart of a relay is an electromagnet (a coil of wire that becomes a temporary magnet when electricity flows) Output of micro-controller is not sufficient to drive the relay directly. Therefore to drive the relay we are using relay driver block by using transistor as switch. A relay is an electrically operated switch. Current flowing through the coil of the relay creates a magnetic field, which attracts a lever and changes the switch contacts. The coil current can be on or off so relays has two switch positions and they are double throw (changeover) switches.

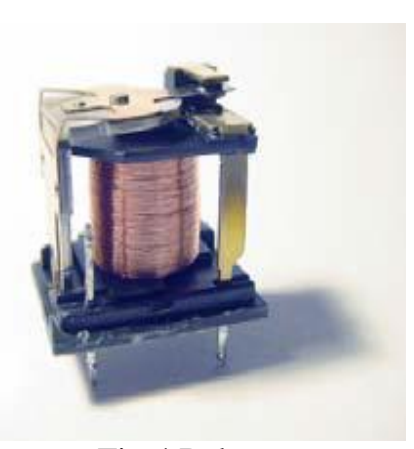

Fig.4.Relay 
Relays allow one circuit to switch a second circuit, which can be completely separate from the first. For example a low voltage battery circuit can use a relay to switch a $230 \mathrm{~V}$ AC mains circuit. There is no electrical connection inside the relay between the two circuits; the link is magnetic and mechanical. The coil of a relay passes a relatively large current, typically $30 \mathrm{~mA}$ for a $12 \mathrm{~V}$ relay, but it can be as much as $100 \mathrm{~mA}$ for relays designed to operate from lower voltages. Most ICs (chips) cannot provide this current and a transistor is usually used to amplify the small current to the larger value required for the relay

\section{SOFTWARE REQUIREMENTS}

\section{VP812 Universal Burner}

AVR Programmer

The programmer should work with all AVRs that can be programmed with AVR ISP interface. The programmer is powered by $5 \mathrm{~V}$ USB power bus, and it is intended for programming AVRs that are running at close to $5 \mathrm{~V}$. VP812 is an excellent USB universal programmer for AVR ICs.

\section{Express PCB}

PCB is a free and open source software suite for electronic design automation - for printed circuit boards layout. PCB was first written by Thomas Nau for an Atari ST in 1990. Initially PCB was not intended to be a professional layout system but as a tool for individuals to do small scale development of hardware.

\section{CONCLUSION}

The press machine is suitable for small scale and medium size industries .A simple an manual operated press machine which is used to punching operation of metal sheet .This project undergoes automation which is controlled by AVR controller .

\section{ACKNOWLEDGMENT}

We would sincerely thank to our guide Miss. S.S.Joshi for her valuable guidance, constant assistance, support endurance and constructive suggestions for the betterment of this platform of research. We also wish to thank all the staff members of the department of E\&TC engineering for helping us directly or indirectly in completing this work successfully.

\section{REFERENCES}

1] Aditya polapragada, sri varsha ,pneumatic Auto feed Punching machine ,international Journal of Engineering Research and Technology (IJERT),vol.1 Issue7,September 2012,ISSN:22780181.

[2] P.M.Pradhan, "Experimental Investigation and Fabrication of Pneumatic punch Innovative Research in Science, Engineering and Technology, Vol. 2, Issue 6, June 2013. 